\title{
On the Differentiable Structure of Meyer Wavelets ${ }^{\star}$
}

\author{
Carlo Cattani ${ }^{1}$ and Luis M. Sánchez Ruiz ${ }^{2}$ \\ ${ }^{1}$ DiFarma, Università di Salerno, Via Ponte Don Melillo 84084 Fisciano (SA)- Italy \\ ccattani@unisa.it \\ ${ }^{2}$ ETSID-Departamento de Matemática Aplicada, Universidad Politécnica de \\ Valencia, 46022 Valencia, Spain \\ Imsr@mat.upv.es
}

\begin{abstract}
In this paper the differential (first order) properties of Meyer wavelets are investigated.
\end{abstract}

Keywords: Meyer Wavelet, Connection coefficients, Refinable integrals.

AMS-Classification - 35A35.

\section{Introduction}

Wavelets have widely been studied from a theoretical point of view for its many interesting properties, mainly related with multiresolution analysis such as generating orthonormal basis in $L_{2}(\mathbb{R})$ as well as for the fact that they have proven to be extremely useful in many applications such as image processing, signal detection, geophysics, medicine or turbulent flows. More mathematically focussed differential equations and even non linear problems have also been studied with wavelets. Very often wavelets are compared with the Fourier basis (harmonic functions), however the basic difference is that the harmonic functions are bounded in the frequency domain (localized in frequency) while wavelets are bounded both in space and in frequency. Nevertheless a major drawback for wavelet theory is the existence of many different families of wavelets, giving some arbitrariness to the whole theory. Among the many families of wavelets the simplest choice is the one based on Haar functions. Despite their simplicity Haar wavelets have proven their suitability for dealing with problems in which piecewise constant functions or functions with sharp discontinuities appear. The scaling function is the box function defined by the characteristic function $\chi_{[0,1]}$ and its Fourier transform, up to constants or phase factor, is a function of the

\footnotetext{
* Work partially supported by Regione Campania under contract "Modelli nonlineari di materiali compositi per applicazioni di nanotecnologia chimica-biomedica", LR 28/5/02 n. 5, Finanziamenti 2003, by Miur under contract "Modelli non Lineari per Applicazioni Tecnologiche e Biomediche di Materiali non Convenzionali", Univ. di Salerno, Processo di internazionalizzazione del sistema universitario, D.M. 5 agosto 2004 n. 262 - ART. 23 and by "Applications from Analysis and Topology" - APLANTOP, Generalitat Valenciana 2005.
} 
type $\frac{\sin (\pi t)}{\pi t}$, also called sinc-function. By exchanging the role of the variable and frequency space, i.e. assuming as Fourier transform the box function and the sinc-function in the space of variable, we can construct the so-called Shannon wavelets 2. In a more general approach they can be derived from the real part of harmonic (complex) wavelets [2] and its scaling function may be obtained by choosing as such a function in the Fourier space such that it satisfies various conditions for a scaling function and then find the wavelet basis. In fact the Haar and Shannon systems reverse the roles of the kind of scaling and wavelet functions. Very recently the connection coefficients both of Shannon wavelets and harmonic wavelets [2 have been explicitly computed at any ordered.

Let us recall that a drawback of the Shannon wavelet in the time domain is its slow decay which has been improved by smoothing the scaling function in the frequency space, e.g. by means of the Meyer scaling function that instead of enjoying a sharp discontinuity, uses a smooth function in order to interpolate between its 1 and 0 values [4. In this paper we study the differentiable structure of Meyer wavelets and, in particular, its connection coefficients in the line of the aformentioned results obtained for the Harmonic [2] and Shannon wavelet.

\section{Meyer's Wavelets}

Meyer's wavelets are defined in a such a way to avoid the slow decay (of compact support frequency domain wavelets) in the space domain. In order to do this one needs a continuous interpolating even function $\nu(\omega)$ defined on $R,[0,1]$-valued which is proportional to $\omega^{n+1}$ (see the interpolating polynomials in table 1).

There follows that the Meyer scaling function is given by [4]

$$
\widehat{\phi}(\omega)= \begin{cases}1 & ,|\omega|<\frac{2 \pi}{3} \\ \cos \left(\frac{\pi}{2} \nu\left(\frac{3}{2 \pi}|\omega|-1\right)\right) & , \frac{2 \pi}{3} \leq|\omega| \leq \frac{4 \pi}{3} \\ 0 & ,|\omega|>\frac{4 \pi}{3}\end{cases}
$$

where $\nu(x)$ (see Table 1) is an interpolating polynomial (see [4]).

Table 1. Interpolating Polynomials

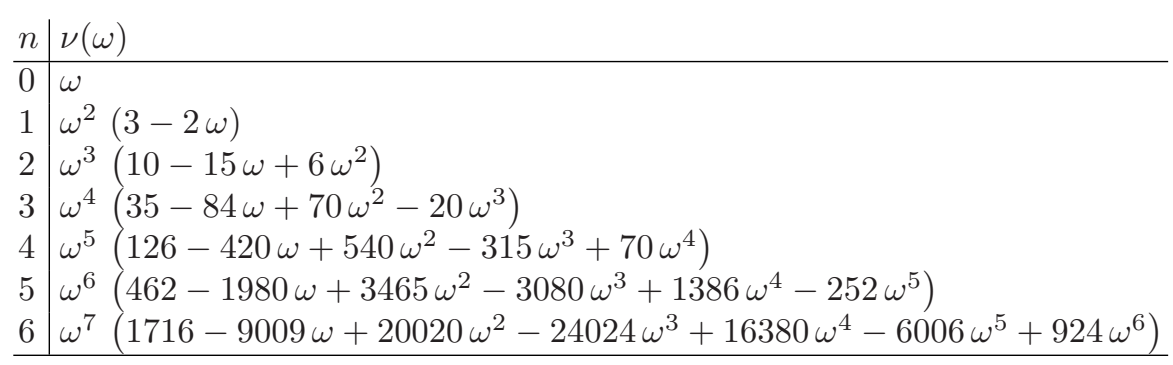


If we define as a characteristic function

$$
\chi(\omega)=\left\{\begin{array}{l}
1, \frac{2}{3} \pi<|\omega|<\frac{4}{3} \pi \\
0, \text { elsewhere }
\end{array}\right.
$$

the scaling function can be written as

$$
\widehat{\phi}(\omega)=\chi\left(\omega+\frac{2}{3} \pi\right)+\frac{1}{2}\left(e^{i \pi \nu\left(\frac{3}{2 \pi}|\omega|-1\right) / 2}+e^{-i \pi \nu\left(\frac{3}{2 \pi}|\omega|-1\right) / 2}\right) \chi(\omega)
$$

By taking into account that, for the properties of the Fourier transform, it is

$$
\widehat{f}(a \omega \pm b)=\frac{1}{a} e^{\mp i b \omega / a} \widehat{f}(\omega / a),
$$

there follows that the dilated and translated instances of the scaling function are

$$
\widehat{\phi}_{k}^{n}(\omega) \stackrel{\text { def }}{=} 2^{n / 2} \phi\left(\widehat{2^{n} t-} k\right)=2^{n / 2} \widehat{\phi}\left(2^{n} \omega-k\right)=2^{n / 2} 2^{-n} e^{-i \omega 2^{-n} k} \widehat{\phi}\left(\omega 2^{-n}\right)
$$

that is

$$
\widehat{\phi}_{k}^{n}(\omega)=2^{-n / 2} e^{-i \omega 2^{-n} k} \widehat{\phi}\left(\omega 2^{-n}\right)
$$

so that the Meyer scaling function at any scale and frequency localization is [4]

$$
\widehat{\phi}_{k}^{n}(\omega)=2^{-n / 2} e^{-i \omega 2^{-n} k} \begin{cases}1 & ,|\omega|<\frac{2^{n+1} \pi}{3} \\ \cos \left(\frac{\pi}{2} \nu\left(\frac{3}{2 \pi}\left|2^{-n} \omega\right|-1\right)\right) & , \frac{2^{n+1} \pi}{3} \leq|\omega| \leq \frac{2^{n+2} \pi}{3} \\ 0 & ,|\omega|>\frac{2^{n+2} \pi}{3}\end{cases}
$$

where $\nu(x)$ is the interpolating polynomial (Table 1). According to (5)-(3) it is

$$
\begin{aligned}
\widehat{\phi}_{k}^{n}(\omega) & =2^{-n / 2} e^{-i \omega 2^{-n} k}\left[\chi\left(\omega 2^{-n}+\frac{2}{3} \pi\right)+\right. \\
& \left.+\frac{1}{2}\left(e^{i \pi \nu\left(\frac{3}{2 \pi}\left|\omega 2^{-n}\right|-1\right) / 2}+e^{-i \pi \nu\left(\frac{3}{2 \pi}\left|\omega 2^{-n}\right|-1\right) / 2}\right) \chi\left(\omega 2^{-n}\right)\right]
\end{aligned}
$$

The scaling function in the time domain is obtained by finding its inverse Fourier transform

$$
\phi(t) \stackrel{\text { def }}{=} \frac{1}{2 \pi} \int_{-\infty}^{\infty} \widehat{\phi}(\omega) e^{i \omega t} \mathrm{~d} \omega=\frac{1}{\pi} \int_{0}^{4 \pi / 3} \widehat{\phi}(\omega) \cos (\omega t) \mathrm{d} \omega
$$

\section{Some Properties of the Characteristic Function}

In general for the characteristic function (2) we can set

$$
\chi_{(a, b]}(\omega)=\left\{\begin{array}{l}
1, a<|\omega| \leq b \\
0, \text { elsewhere }
\end{array}\right.
$$


for which, assuming $a \leq b, c \leq d, h>0, k>0$, the following properties

$$
\begin{cases}\chi_{(a, b]}(h \omega \pm k) & =\chi_{\left(\frac{a}{h} \mp k, \frac{b}{h} \mp k\right]}(\omega) \\ \chi_{(a, b]}(-h \omega) & =\chi_{(a, b]}(h \omega) \\ \chi_{(a, b]}(\omega)+\chi_{(c, d]}(\omega) & =\chi_{(a, b] \cup(c, d]}(\omega)+\chi_{(a, b] \cap(c, d]}(\omega) \\ \chi_{(a, b]}(\omega) \chi_{(c, d]}(\omega) & =\chi_{(a, b] \cap(c, d]}(\omega) \\ \chi_{(a+s, b+s]}(\omega) \chi_{(c+s, d+s]}(\omega) & =\chi_{(a, b]}(\omega) \chi_{(c, d]}(\omega)=\chi_{(a, b] \cap(c, d]}(\omega)\end{cases}
$$

hold. According to the previous equations, the characteristic function (9) on any interval $(a, b]$ can be reduced to the basic function (2):

$$
\chi_{(a, b]}(\omega)=\chi\left(\frac{2 \pi}{3(b-a)} \omega-(b-2 a)\right), \quad b>a .
$$

Analogously, according to (10) 1 and (2), we can generalize the basic function (2) to any interval being

$$
\chi(h \omega \pm k)=\chi_{\left(\frac{2 \pi}{3 h} \mp k, \frac{4 \pi}{3 h} \mp k\right]}(\omega) .
$$

The product of the characteristic functions, enjoys some nice properties:

Lemma 1. For the product of the characteristic functions we have:

$$
\chi\left(2^{-m} \omega+\frac{2}{3} \pi\right) \chi\left(2^{-n} \omega\right)=\chi_{\left(\frac{2 \pi}{3} 2^{m}-\frac{2}{3} \pi, \frac{4 \pi}{3} 2^{m}-\frac{2}{3} \pi\right] \cap\left(\frac{2 \pi}{3} 2^{n}, \frac{4 \pi}{3} 2^{n}\right]}(\omega)
$$

with

$$
\begin{aligned}
& \left(\frac{2 \pi}{3} 2^{m}-\frac{2}{3} \pi, \frac{4 \pi}{3} 2^{m}-\frac{2}{3} \pi\right] \cap\left(\frac{2 \pi}{3} 2^{n}, \frac{4 \pi}{3} 2^{n}\right]= \\
& =\left\{\begin{array}{l}
\emptyset \Longleftrightarrow 2^{m}<\frac{1}{2}+2^{n-1} \vee 2^{m}>1+2^{n+1} \\
\left(\frac{4 \pi}{3} 2^{m}-\frac{2}{3} \pi, \frac{2 \pi}{3} 2^{n}\right] \Longleftrightarrow 2^{n-1}+\frac{1}{2}<2^{m}<2^{n}+\frac{1}{2} \\
\left(\frac{2 \pi}{3} 2^{n}, \frac{4 \pi}{3} 2^{n}\right] \Longleftrightarrow 2^{n}+\frac{1}{2}<2^{m}<2^{n}+1 \\
\left(\frac{2 \pi}{3} 2^{m}-\frac{2}{3} \pi, \frac{4 \pi}{3} 2^{n}\right] \Longleftrightarrow 2^{n}+1<2^{m}<2^{n+1}+1
\end{array}\right.
\end{aligned}
$$

being $\chi_{\emptyset}(\omega)=0$

Analogously it can be easily shown that

Lemma 2. For the product of the characteristic functions we have:

$$
\chi\left(2^{m} \omega+\frac{2}{3} \pi\right) \chi\left(2^{n} \omega\right)=\chi_{\left(\frac{2 \pi}{3} 2^{-m}-\frac{2}{3} \pi, \frac{4 \pi}{3} 2^{-m}-\frac{2}{3} \pi\right] \cap\left(\frac{2 \pi}{3} 2^{-n}, \frac{4 \pi}{3} 2^{-n}\right]}(\omega)
$$


From the above there follows, in particular

$$
\left\{\begin{array}{l}
\chi\left(2^{n} \omega+\frac{2}{3} \pi\right) \chi\left(2^{n-1} \omega\right)=0 \\
\chi\left(2^{n-1} \omega+\frac{2}{3} \pi\right) \chi\left(2^{n} \omega\right)=0
\end{array}\right.
$$

Another group of values of characteristic functions useful for the following computations are given by the following lemmas

Lemma 3. For the following products of the characteristic functions we have:

$$
\begin{aligned}
& \chi\left(2^{-m} \omega\right) \chi\left(2^{-n} \omega\right)=\chi_{\left(\frac{2 \pi}{3} 2^{m}, \frac{4 \pi}{3} 2^{m}\right] \cap\left(\frac{2 \pi}{3} 2^{n}, \frac{4 \pi}{3} 2^{n}\right]}(\omega) \\
& \chi\left(2^{m} \omega\right) \chi\left(2^{n} \omega\right)=\chi_{\left(\frac{2 \pi}{3} 2^{-m}, \frac{4 \pi}{3} 2^{-m}\right] \cap\left(\frac{2 \pi}{3} 2^{-n}, \frac{4 \pi}{3} 2^{-n}\right]}(\omega)
\end{aligned}
$$

Taking into account the previous lemmas and (10) 5 we have also

Corollary 1. According to lemma 3 it is

$$
\chi\left(2^{m} \omega+\frac{2}{3} \pi\right) \chi\left(2^{n} \omega+\frac{2}{3} \pi\right)=\chi\left(2^{m} \omega\right) \chi\left(2^{n} \omega\right),
$$

and, in particular,

$$
\left\{\begin{array}{l}
\chi\left(2^{n} \omega+\frac{2}{3} \pi\right) \chi\left(2^{n-1} \omega+\frac{2}{3} \pi\right)=0 \\
\chi\left(2^{n} \omega\right) \chi\left(2^{n-1} \omega\right)=0
\end{array}\right.
$$

\section{First Order Meyer Wavelet}

If we take as interpolating function (Table 1) the linear one $\nu(\omega)=\omega$, we get from (6),

$$
\widehat{\phi}_{k}^{n}(\omega)=2^{-n / 2} e^{-i \omega 2^{-n} k} \begin{cases}1 & ,|\omega|<\frac{2^{n+1} \pi}{3} \\ \sin \left(\frac{3}{4}\left|2^{-n} \omega\right|\right) & , \frac{2^{n+1} \pi}{3} \leq|\omega| \leq \frac{2^{n+2} \pi}{3} \\ 0 & ,|\omega|>\frac{2^{n+2} \pi}{3}\end{cases}
$$

that is

$$
\widehat{\phi}_{k}^{n}(\omega)=2^{-n / 2} e^{-i \omega 2^{-n} k}\left[\chi\left(2^{-n} \omega+\frac{2}{3} \pi\right)+\sin \left(2^{-n-2} 3|\omega|\right) \chi\left(2^{-n} \omega\right)\right]
$$

From equation (19) it immediately follows that for $k=0$ the scaling functions $\widehat{\phi}_{0}^{n}(\omega)$ are real functions while for $k \neq 0$ the functions $\widehat{\phi}_{k}^{n}(\omega)$ have also a nontrivial complex part (see Fig. 11). Moreover, the functions $\widehat{\phi}_{0}^{n}(\omega)$ are orthonormal functions with respect to the inner product on $L^{2}(\mathbb{R})$ : 

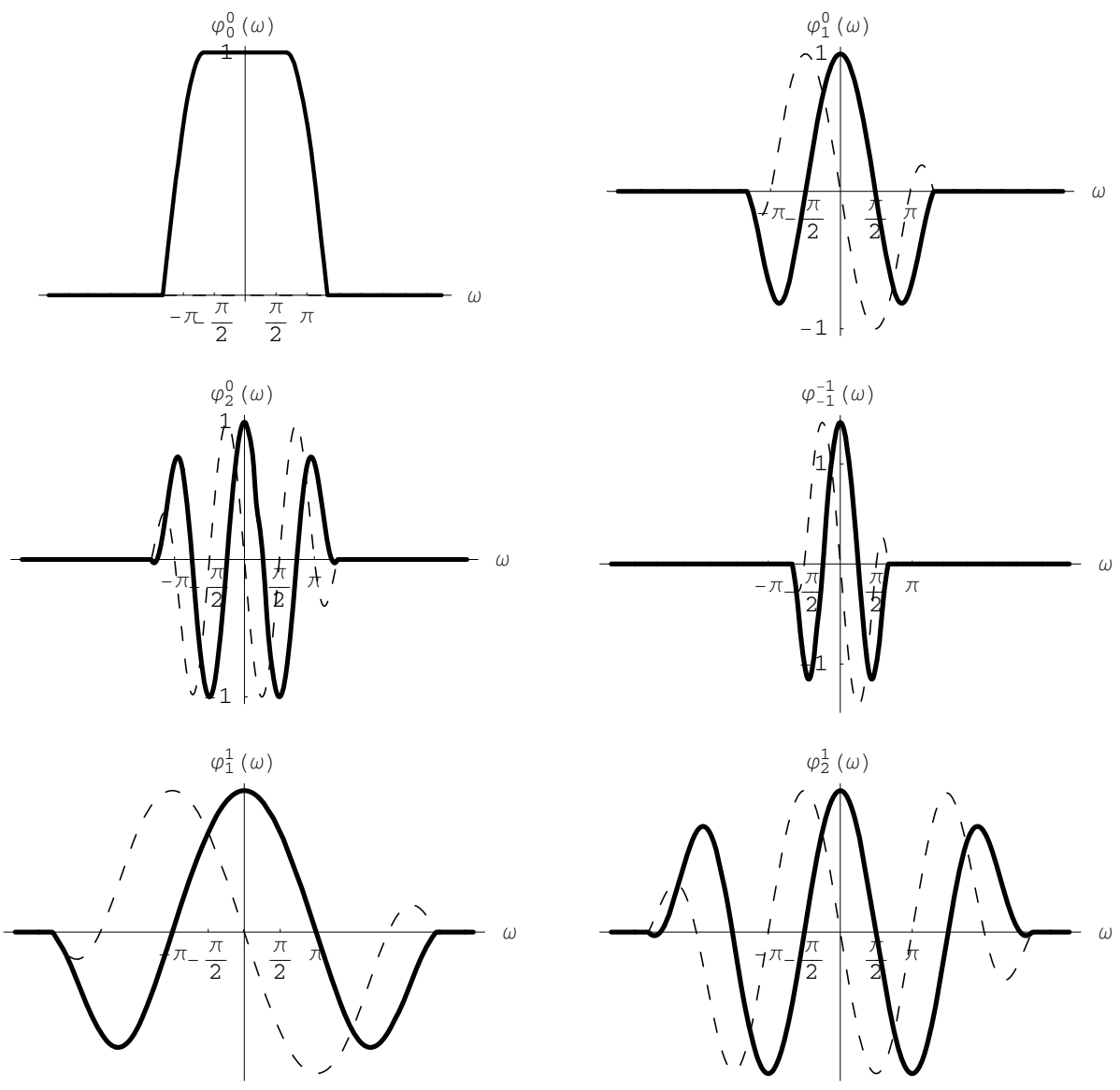

Fig. 1. The Meyer scaling function in frequency domain (plain the real part)

$$
\langle f, g\rangle \stackrel{\text { def }}{=} \int_{-\infty}^{\infty} f(t) \overline{g(t)} \mathrm{d} t
$$

with $f(t), g(t)$, in $L^{2}(\mathbb{R})$ and the bar stands for the complex conjugate. By taking into account the Parseval equality it is

$$
\langle f, g\rangle=\frac{1}{2 \pi} \int_{-\infty}^{\infty} \widehat{f}(\omega) \bar{g}(\omega) \mathrm{d} \omega=\frac{1}{2 \pi}\langle\widehat{f}, \widehat{g}\rangle .
$$

In the Fourier domain, it is possible to show that 
Theorem 1. The scaling functions $\left\{\phi_{k}^{0}(t)\right\}$ are orthonormal (see e.g. [4])

$$
\left\langle\phi_{k}^{0}(t), \phi_{h}^{0}(t)\right\rangle=\delta_{k h} .
$$

Proof: It is enought to show that, due to (21),

$$
\frac{1}{2 \pi}\left\langle\widehat{\phi}_{k}^{0}(\omega), \widehat{\phi}_{h}^{0}(\omega)\right\rangle=\delta_{k h}
$$

From equation (19) and taking into account the definition (20) of the inner product it is

$$
\begin{aligned}
\left\langle\widehat{\phi}_{k}^{0}(\omega), \widehat{\phi}_{h}^{0}(\omega)\right\rangle & =\int_{-\infty}^{\infty} e^{-i \omega k}\left[\chi\left(\omega+\frac{2}{3} \pi\right)+\sin \left(\frac{3}{4}|\omega|\right) \chi(\omega)\right] \times \\
& \times e^{i \omega h}\left[\chi\left(\omega+\frac{2}{3} \pi\right)+\sin \left(\frac{3}{4}|\omega|\right) \chi(\omega)\right] \mathrm{d} \omega
\end{aligned}
$$

Since the compact support of the characteristic functions are disjoint: $\chi(\omega+$ $\left.\frac{2}{3} \pi\right) \chi(\omega)=0$, we get

$$
\left\langle\widehat{\phi}_{k}^{0}(\omega), \widehat{\phi}_{h}^{0}(\omega)\right\rangle=\int_{-\infty}^{\infty} e^{-i \omega(k-h)}\left[\chi\left(\omega+\frac{2}{3} \pi\right)+\sin ^{2}\left(\frac{3}{4}|\omega|\right) \chi(\omega)\right] \mathrm{d} \omega
$$

i.e. taking into account the definition of the characteristic functions

$$
\begin{aligned}
& \left\langle\widehat{\phi}_{k}^{0}(\omega), \widehat{\phi}_{h}^{0}(\omega)\right\rangle=\int_{-2 \pi / 3}^{2 \pi / 3} e^{-i \omega(k-h)} \mathrm{d} \omega+ \\
& +\int_{-4 \pi / 3}^{-2 \pi / 3} e^{-i \omega(k-h)} \sin ^{2}\left(\frac{3}{4}|\omega|\right) \mathrm{d} \omega+\int_{2 \pi / 3}^{4 \pi / 3} e^{-i \omega(k-h)} \sin ^{2}\left(\frac{3}{4}|\omega|\right) \mathrm{d} \omega
\end{aligned}
$$

Thus when $k=h$,

$$
\begin{aligned}
\left\langle\widehat{\phi}_{k}^{0}(\omega), \widehat{\phi}_{k}^{0}(\omega)\right\rangle & =\int_{-2 \pi / 3}^{2 \pi / 3} \mathrm{~d} \omega+\int_{-4 \pi / 3}^{-2 \pi / 3} \sin ^{2}\left(\frac{3}{4} \omega\right) \mathrm{d} \omega+\int_{2 \pi / 3}^{4 \pi / 3} \sin ^{2}\left(\frac{3}{4} \omega\right) \mathrm{d} \omega \\
& =\frac{4}{3} \pi+\frac{\pi}{3}+\frac{\pi}{3}=2 \pi
\end{aligned}
$$

when $k \neq h$, let say $k=h+n$, it is

$$
\left\langle\widehat{\phi}_{k}^{0}(\omega), \widehat{\phi}_{h}^{0}(\omega)\right\rangle=\frac{1}{n} \sin \left(\frac{2}{3} n \pi\right) \frac{9+18 \cos \left(\frac{2}{3} n \pi\right)}{9-4 n^{2}}=0 .
$$

There follows that

$$
\left\langle\widehat{\phi}_{k}^{0}(\omega), \widehat{\phi}_{h}^{0}(\omega)\right\rangle= \begin{cases}2 \pi, & k=h \\ 0 & , k \neq h\end{cases}
$$


The Meyer wavelet in the Fourier domain is [4]

$$
\widehat{\psi}(\omega)=-e^{-i \omega / 2}[\widehat{\phi}(\omega-2 \pi)+\widehat{\phi}(\omega+2 \pi)] \widehat{\phi}(\omega / 2)
$$

and, according to (4),

$$
\widehat{\psi}(\omega)=-e^{-i \omega / 2}\left[e^{2 \pi i \omega}+e^{-2 \pi i \omega}\right] \widehat{\phi}(\omega) \widehat{\phi}(\omega / 2)
$$

i.e.

$$
\widehat{\psi}(\omega)=-2 e^{-i \omega / 2} \cos (2 \pi \omega) \widehat{\phi}(\omega) \widehat{\phi}(\omega / 2)
$$

From (22) we can easily derive the dilated and translated instances

$$
\widehat{\psi}_{k}^{n}(\omega)=-2^{-n / 2+1} e^{-i 2^{-n} \omega(k+1 / 2)} \cos \left(2^{-n+1} \pi \omega\right) 2^{n / 2} \widehat{\phi}_{0}^{-n}(\omega) 2^{(n-1) / 2} \widehat{\phi}_{0}^{-n+1}(\omega)
$$

i.e.

$$
\widehat{\psi}_{k}^{n}(\omega)=-2^{(n+1) / 2} e^{-i 2^{-n} \omega(k+1 / 2)} \cos \left(2^{-n+1} \pi \omega\right) \widehat{\phi}_{0}^{-n}(\omega) \widehat{\phi}_{0}^{-n+1}(\omega)
$$

From this definition we can easily prove that Meyer wavelets are orthonormal functions.

\section{References}

1. D.E. Newland, "Harmonic wavelet analysis", Proc.R.Soc.Lond. A, 443, (1993) 203-222.

2. C.Cattani, "Harmonic Wavelets towards Solution of Nonlinear PDE", Computers and Mathematics with Applications, 50 (2005), 1191-1210.

3. H. Mouri and H.Kubotani, "Real-valued harmonic wavelets", Phys.Lett. A, 201, (1995) 53-60.

4. I. Daubechies, Ten Lectures on wavelets. SIAM, Philadelphia, PA, (1992). 